\title{
A Formal Framework of Conversational Goals Based on Strategic Reasoning
}

\author{
Mohamed Mbarki ${ }^{1}$, Jamal Bentahar ${ }^{2}$, and Bernard Moulin ${ }^{1}$ \\ ${ }^{1}$ Laval University, Department of Computer Science and Software Engineering, \\ Canada \\ mohamed.mbarki.1@ulaval.ca, bernard.moulin@ift.ulaval.ca \\ ${ }^{2}$ Concordia University, Concordia Institute for Information Systems Engineering \\ (CIISE), Canada \\ bentahar@ciise. concordia.ca
}

\begin{abstract}
In this paper, we propose a formal framework of strategic reasoning that agents use when interacting in a dialogue and trying to achieve their conversational goals. Such argumentation-based reasoning enables agents to generate a set of strategic goals depending on a set of constraints. Strategic goals are sub-goals, which are necessary to achieve a conversational goal. Some of these sub-goals are generated at the beginning of the interaction and they can be canceled or substituted by alternatives during the dialogue. An original characteristic of this framework is that it enables agents to persist in the achievement of their goals by considering alternatives and calculating the feasibility of each sub-goal. The set of constraints as well as sub-goals can be set at the beginning or during the dialogue.
\end{abstract}

\section{Introduction}

In multi-agent systems, agents are designed to perform particular tasks. Autonomy and intelligence are the most important characteristics of these agents, by which they should be able to generate and achieve their own goals. In the modern research into multi-agent systems, agents are equipped with reasoning capabilities expressed in computational logics [6]7. Each agent has its own domain and certain goals to achieve what we call operational goals. To achieve their operational goals, agents often have to interact with each other. To participate in such interactions, agents need to create what we call conversational goals. Consequently, conversational goals must satisfy a set of operational constraints. Conversational goals must also satisfy a set of conversational constraints which are related to the conversational context. Time and budget constraints are examples of operational constraints, and respecting the religious and ideological beliefs of the addressee is an example of conversational constraints. The operational and conversational constraints may be revised during the dialogue. It is thus imperative to take into account these two types of constraints during the generation and the realization of conversational goals. Such constraints have been neglected in the most recent approaches based on goal modeling (e.g. [15]). 
In this paper we present a formal framework to reason about conversational goals using sub-goals, prospective alternatives and constraints associated with each goal. The idea is to give agents the possibility to modify or reject their sub-goals and revise the set of constraints they decide to satisfy. This enables agents to decide about how to achieve their conversational goals considering the sub-goals and the sets of constraints to satisfy. Alternative sub-goals can also be considered in order to enable agents to persist in the achievement of their conversational goals, even in the case of the cancellation of one of them. Although the notion of persistence is important to ensure the conversation success, it has not been introduced in current communication models (e.g. [1], 3], [4] and [5]). In this context, the achievement of a conversational goal will depend on the realization of its sub-goals by taking into account operational and conversational constraints.

In this paper, we illustrate our model using an example of negotiation (of cars) between two agents. We suppose that an agent $A g_{1}$ tries to convince another agent $A g_{2}$ to buy a car. In this example, the seller's conversational goal, denoted $B$, corresponds to the objective of the conversation which is the sale of a car.

This paper is organized as follows. In Section 2, we introduce the agent model that defined the strategic reasoning in terms of strategic goals, constraints and possible alternatives. In Section 3. we present an argumentation-based reasoning which enables an agent to generate the sub-goals that are necessary for the achievement of its conversational goal. Before concluding, we present in Section 4 a formal framework which enables agents to persist in the trying to achieve their goals by the use of alternatives, and we show how agents can compute the feasibility of their sub-goals.

\section{Agent Theory}

Based on its mental states and other kinds of information (essentially provided by the social context and the conversational context), an agent can have a global vision to achieve its conversational goal. This vision is considered as a strategy that enables agents to generate a set of sub-goals, that we call strategic goals, which depend on a set of constraints. These strategic goals are selected and organized in order to achieve the conversational goal. Each strategic goal can be decomposed into a set of sub-goals which can themselves be decomposed recursively until elementary goals are reached. We call the generation of strategic goals: strategic reasoning. The choice of strategic goals is detailed in Section 3 ,

A strategic goal can have one or more alternatives, and the replacement of this goal by one of its alternatives enables an agent to achieve the same conversational goal, while satisfying different constraints. The subset of constraints to be satisfied and the subset of sub-goals to be realized in order to achieve the conversational goal determine the adopted strategy. Strategies are dynamic by nature and agents should adjust the adopted strategy while the conversation progresses. This can be achieved by taking into account the new constraints that can appear during the conversation. In this case, the new constraints to be 
satisfied should be consistent with the initial sub-set of constraints and criterions that need to be satisfied.

In our negotiation example, the seller agent can choose the strategy according to which the conversational goal $B$, which is the sale of a car, can be decomposed into three strategic goals $B_{1}, B_{2}$ and $B_{3}$. These sub-goals may be defined as follows: $B_{1}=$ "know how much the buyer would like to invest in the purchase of a car as well as his preferences", $B_{2}=$ "propose a car which may interest the buyer" and $B_{3}=$ "convince the buyer to accept this proposition".

The goal $B_{1}$ can be decomposed into two sub-goals $B_{11}$ and $B_{12}$. The goal $B_{3}$ can also be decomposed into two sub-goals $B_{31}$ and $B_{32}$. These new sub-goals are defined as follows: $B_{11}=$ "know which model of cars preferred by the buyer", $B_{12}=$ "know how much the buyer would like to invest", $B_{31}=$ "convince the buyer that the price of the proposed car is reasonable" and $B_{32}=$ "convince the buyer that the proposed car consumes like small cars on the long distances".

In our example, we suppose that the seller is unable to convince the buyer to accept its offer (i.e. the seller is unable to achieve goal $B_{3}$ by using elementary actions) because a new conversational constraint occurs. In this case, the seller agent must persist in trying to achieve its goal by considering an alternative to goal $B_{2}$. For example, the seller agent may propose another car which may satisfy the new buyer's interest, and this new bid will be an alternative goal for $B_{2}$, named $B_{2}^{\prime}$.

Moreover, if the seller agent finds during the dialogue that the buyer agent is not interested by the car gas consumption, then it may suggest an alternative to the strategic goal $B_{32}$, denoted $B_{32}^{\prime}$. For example, the seller may try to convince the buyer that the spare parts for the proposed car are available and not expensive. The strategic goals $B_{2}^{\prime}$ and $B_{32}^{\prime}$ may be defined as follows: $B_{2}^{\prime}=$ "propose another car which may satisfy a new buyer's interest" and $B_{32}^{\prime}=$ "convince the buyer that spare parts for this car are available and not expensive".

Our framework uses knowledge, goals and constraints. We introduce a formal language to manipulate these elements:

Definition 1 (knowledge, goal and constraint formulas). Let $\mathcal{L}$ with typical element $\phi$ be a propositional language with negation and conjunction. The knowledge formulas $\mathcal{L}_{\Gamma}$ with typical element $p$, the goal formulas $\mathcal{L}_{\mathcal{G}}$ with typical element $B$ and the constraint formulas $\mathcal{L}_{\mathcal{C}}$ with typical element $c$ are defined as follows.

- if $\phi \in \mathcal{L}$, then $\Gamma \phi \in \mathcal{L}_{\Gamma}, \mathcal{G} \phi \in \mathcal{L}_{\mathcal{G}}$ and $\mathcal{C} \phi \in \mathcal{L}_{\mathcal{C}}$

- if $p_{1}, p_{2} \in \mathcal{L}_{\Gamma}, B_{1}, B_{2} \in \mathcal{L}_{\mathcal{G}}$ and $c_{1}, c_{2} \in \mathcal{L}_{\mathcal{C}}$ then $\neg p_{1}, p_{1} \wedge p_{2} \in \mathcal{L}_{\Gamma}, \neg B_{1}, B_{1} \wedge$ $B_{2} \in \mathcal{L}_{\mathcal{G}}$ and $\neg c_{1}, c_{1} \wedge c_{2} \in \mathcal{L}_{\mathcal{C}}$.

\section{Argumentation-Based Strategic Goals}

In our framework, the adoption of a set of strategic goals by an agent must be supported by internal arguments. For this reason, we define an explanatory argument concept, inspired by Amgoud and Kaci [1], and a realization argument 
concept. A given goal may be supported by these two types of arguments. The explanatory arguments justify the feasibility of the strategic goals in terms of beliefs. In contrast, the realization arguments determine the set of strategic goals necessary to achieve a conversational or a strategic goa 1 . We define in this section an argumentation-driven framework to generate the set of operational and conversational constraints related to a strategic goal. We also define the generation of strategic goals and their alternatives in order to achieve a conversational goal, while respecting the set of constraints related to this goal. In the rest of the paper, $\Gamma$ indicates a possibly inconsistent knowledge base with no deductive closure, $\mathcal{C}$ indicates the set of constraints and $\mathcal{G}$ indicates a consistent set of goals. In addition we define two relations $\vdash_{\Gamma}$ and $\vdash_{\mathcal{C}}$, the former stands for classical inference and the latter stands for the need to achieve some sub-goals to achieve another goal.

Definition 2 (Explanatory Argument). An explanatory argument of an agent $A g$ is a pair $(H, B)$ where $B$ indicates the $A g$ 's goal and it is expressed as a formula in $\mathcal{L}_{G}$ and $H$ is a subset of $\Gamma$ such that: $\left.i\right) H$ is consistent, ii) $H \vdash_{\Gamma} B$, and iii) $H$ is minimal (there is no subset of $H$ which satisfies $i$ and $i i)$. An explanatory argument satisfying $i$ and $i i$ but not necessarily iii is called a non necessarily minimal argument. $H$ is called the support of the argument which justifies the feasibility of the goal $B$ in terms of beliefs.

Definition 3 (Realization Argument). A realization argument of an agent $A g$ is a triplet $(G, B, C)$ where $G$ is a finite set of $A g$ 's strategic goals $(G \subseteq \mathcal{G})$, $B$ indicates that $A g$ has the goal $B(B \in \mathcal{G})$, and $C$ is a finite set of constraints $(C \subseteq \mathcal{C})$ such that $: i)$ all the goals of $G$ are supported by explanatory arguments, ii) $G$ is consistent, iii) $G \vdash_{\mathcal{C}} B$, and $\left.i v\right) G$ is minimal (there is no subset of $G$ which satisfies $i$, ii and iii). $G$ is called the support of the argument which justifies the choice of the set of the strategic goals necessary for the realization of the goal $B$.

$G$ represents the strategic goals that $A g$ can use to achieve the goal $B$ while satisfying a set of constraints $C$. Hence, the problem is: given an agent's goal $B$ and a set of constraints $C$, what is the set of strategic goals $G$ that need to be realized in order to achieve the goal $B$. By using explanatory and realization arguments, we can define the strategic goals and their possible alternatives in order to achieve a given conversational goal.

Definition 4 (Strategic Goal). Let $G$ be a finite set of $A g$ 's goals, $B$ be an $A g$ 's conversational or strategic goal, $C$ be a finite set of constraints, and $\operatorname{Str} G(B)$ be a set of strategic goals (i.e. sub-goals) necessary to realize $B . B^{\prime}$ is a strategic goal of $B\left(B^{\prime} \in \operatorname{Str} G(B)\right)$ iff there is a realization argument $(G, B, C)$ such that: $G \subseteq \mathcal{G}$ and $B^{\prime} \in G$.

The fact that $G$ is minimal makes $B^{\prime}$ necessary for the realization of $B$. However, $B^{\prime}$ may be substituted by another sub-goal called alternative goal.

\footnotetext{
${ }^{1}$ Agents can have plans specified by a set of rules, which enable them to select or revise their realization arguments.
} 
Definition 5 (Alternative Goal). Let $G$ be a finite set of Ag's goals, $B$ be an $A g$ 's conversational or strategic goal, $B_{i}$ be a strategic goal necessary for the realization of $B, C$ be a finite set of constraints associated to $B, C_{i}$ be a finite set of constraints associated to $B_{i}$, and $\operatorname{Alt} G\left(B_{i} / B\right)$ be a set of alternative goals of a strategic goal $B_{i} . B_{j}$ is an alternative goal of $B_{i}\left(B_{j} \in \operatorname{Alt} G\left(B_{i} / B\right)\right)$ iff:

1. $(G, B, C)$ is a realization argument such that $B_{i} \in G$;

2. $\left(G \cup\left\{B_{j}\right\}-\left\{B_{i}\right\}, B, C \cup C_{j}-C_{i}\right)$ is also a realization argument of $B$ such that $C_{j}$ is the finite set of constraints associated to $B_{j}$.

Proposition 1. Let $B_{j}$ be an alternative goal of a strategic goal $B_{i}$ associated to a strategic or conversational goal $B . B_{j}$ is also a strategic goal of $B$.

Proof. According to the second condition of Definition 5 , there is a realization argument $\left(G, B, C \cup C_{j}-C_{i}\right)$ of $B$ such that $B_{j} \in G$ and $C_{i}, C_{j}$ are respectively the sets of constraints associated to $B_{i}$ and $B_{j}$. Consequently and according to Definition $4, B_{j}$ is a strategic goal of $B$.

\section{Constraint Generation and Goal Feasibility}

Strategic reasoning enables agents to generate the strategic goals that ensure the realization of the conversational goal while respecting, at each step of the dialogue, the set of constraints associated with this goal. The initial operational and conversational constraints of a conversational goal are not generated by the agent which is committed to achieve this goal, but they are given by another agent (or the user) at the moment of the commitment. However, the initial constraints related to a strategic goal are calculated when this goal is generated. New constraints related to the strategic goals may be added during the dialogue progress. In this case, the set of constraints of the strategic goal that the agent tries to satisfy in the current step of the dialogue must be revised. This new set of constraints must be satisfied, otherwise the goal related to this set may not be achieved. With respect to the satisfaction of the set of constraints related to the conversational goal, there are two possible cases. If the new set of constraints related to the strategic goal is consistent with the set of constraints related to the conversational goal, the agent can keep the same strategy. In the second case, the agent must adjust its strategy by replacing the strategic goal by one of its alternatives. If some inconsistency persists, the agent must change its strategy completely. The dialogue terminates if the agent does not succeed in finding a new strategy, and we say that the realization of the conversational goal failed.

In our model, the set of agent's goals is represented by a tree in which the root represents the conversational goal (represented by square in Fig 11), the nodes represent strategic goals (also represented by squares), and the leaves represent elementary goals (represented by circles), which can be achieved by performing speech acts. This tree is built progressively while the dialogue progresses. To simplify the notation, an agent's conversational goal will be denoted by $B$ and its strategic goals will be denoted by $B_{i j}$. The principal idea of the goals decomposition is that for each goal (conversational or strategic) we have: 


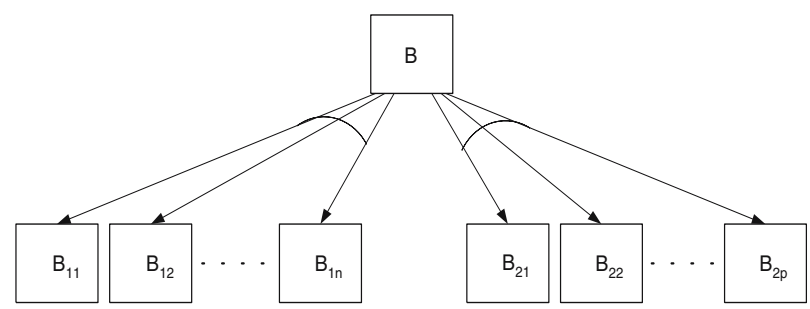

Fig. 1. Example of decomposition of a conversational or strategic goal

1. If the goal is elementary, then the agent tries to satisfy it by using tactical reasoning. This reasoning enables agents to choose the most relevant speech act in order to achieve an elementary goal. More details about this type of reasoning can be found in [2].

2. If the goal is decomposable, then the agent must determine, using an argumentative process, the sub-goals to achieve in order to achieve the initial goal. For each sub-goal, the agent may have several alternatives having different constraints. The achievement of these alternatives can provide the same result as the initial sub-goal. The set of strategic goals which may be used to achieve the same task are connected by a concave arc, as indicated in Fig.1. In this figure, the goal $B$ may be decomposed into two goals $B_{11}$ and $B_{21}$. For the goal $B_{11}$, we can have several alternatives $\left(B_{12}, \ldots, B_{1 n}\right)$ and its realization requires its achievement or the achievement of one of its alternatives (there is thus a disjunction). In the same way, the realization of $B_{21}$ requires its achievement or the achievement of one of its alternatives: $B_{22}, \ldots$, or $B_{2 p}$. Finally, the realization of the initial goal $B$ requires the achievement of a goal from the set $\left\{B_{11}, B_{12}, \ldots, B_{1 n}\right\}$ and a goal from the set $\left\{B_{21}, B_{22}, \ldots, B_{2 p}\right\}$ (there is thus a conjunction).

In a general way, the generation of the set of constraints related to a set of strategic goals is defined as follows.

Definition 6 (Constraint Generation). Let $B$ be a conversational or strategic goal and $\mathcal{E}$ be a function associating elementary goals to a set of constraints. The constraints associated to the set of the strategic goals $(\operatorname{Str} G(B))$ of the goal $B$ is given by the function $\mathcal{C}$ tr which is defined in Table 1.

In Definition [6, the function $\mathcal{C}$ tr takes as an argument a set of goals (or a set of graphs) and returns a set of subsets of constraints representing the set of possible scenarios (i.e. each subset represents a scenario that an agent may consider). $\alpha$ and $\beta$ are two sets of constraints. $B(1)$ represents the first strategic goal of the goal $B$ and its possible alternatives. $\overline{B(1)}$ represents the remainder of the goals in the tree representing the goal $B$. In a general way, $B(i)$ represents the set including the $\mathrm{i}$-th strategic goal of the goal $B$ and its possible alternatives and $\overline{B(i)}$ represents the remainder of the goals of the tree representing the goal $B$. For example, in Fig 1 we have: $B(1)=\left\{B_{11}, B_{12}, \ldots, B_{1 n}\right\}$ and 
Table 1. Constraint Generation Function

\begin{tabular}{ll}
$\mathcal{C} \operatorname{tr}(\emptyset)$ & $=\{\emptyset\}$ \\
$\mathcal{C} \operatorname{tr}(\{B\})$ & $=\mathcal{E}(B)$ if $B$ is elementary \\
$\mathcal{C} \operatorname{tr}(\{B\})$ & $=\left\{\bigcup_{\alpha \in \mathcal{C} \operatorname{tr}(\{B(1)\}) \quad \beta \in \mathcal{C} \operatorname{tr}(\{\overline{B(1)}\})} \alpha \cup \beta\right\}$ if $B$ is not elementary \\
$\mathcal{C} \operatorname{tr}\left(\left\{B_{1}, B_{2}, \ldots, B_{n}\right\}\right)$ & $=\mathcal{C} \operatorname{tr}\left(\left\{B_{1}\right\}\right) \cup \mathcal{C} \operatorname{tr}\left(\left\{B_{2}\right\}\right) \cup \ldots \cup \mathcal{C} \operatorname{tr}\left(\left\{B_{n}\right\}\right)$ \\
\hline
\end{tabular}

$\overline{B(1)}=\left\{B_{21}, B_{22}, \ldots, B_{2 p}\right\}$. Furthermore, we consider that the constraints related to an elementary goal are generated from the speech act performed to achieve this goal. For example, for the speech act: "I sell you my watch for 5 dollars", the function $\mathcal{E}$ provides the set which contains the constraint: " the price is equal to 5 dollars".

Definition 7 (Goal Feasibility). Let $B$ be a conversational goal and $C(B)$ be the set of its initial constraints. We say that $B$ is a feasible goal or realizable if there is an element $C_{i}$ of $\mathcal{C} \operatorname{tr}(\{B\})$ such that: $C(B) \cup C_{i}$ is consistent.

According to Definition 7 , a conversational goal $B$ cannot be achieved if there is no subset of constraints of the set $\mathcal{C} \operatorname{tr}(\{B\})$ which is consistent with the set $C(B)$ (set of constraints associated with the agent conversational goal). In other words, an agent that is able to satisfy the constraints which appear during the dialogue would be able to achieve its conversational goal.

In our example of car negotiation (Section 2), the conversational goal is $B$, the set of the strategic goals of $B$ is $\operatorname{Str} G(B)=\left\{B_{1}, B_{2}, B_{3}, B_{2}^{\prime}\right\}$, where $B_{2}$ and $B_{2}^{\prime}$ are two alternative goals $\left(B_{2}^{\prime} \in \operatorname{Alt} G\left(B_{2} / B\right)\right)$, the set of the strategic goals of $B_{1}$ is $\operatorname{Str} G\left(B_{1}\right)=\left\{B_{11}, B_{12}\right\}$, and the set of strategic goals of $B_{3}$ is $\operatorname{Str} G\left(B_{3}\right)=\left\{B_{31}, B_{32}, B_{32}^{\prime}\right\}$, where $B_{32}$ and $B_{32}^{\prime}$ are also two alternative goals $\left(B_{32}^{\prime} \in \operatorname{Alt} G\left(B_{32} / B\right)\right)$. The set of these goals is presented by a tree in Fig 2

We also suppose that the set of initial operational and conversational constraints that the agent decides to satisfy is: $C(B)=\left\{c, c^{\prime}\right\}, \mathcal{E}\left(B_{11}\right)=\left\{\left\{c_{11}\right\}\right\}$,

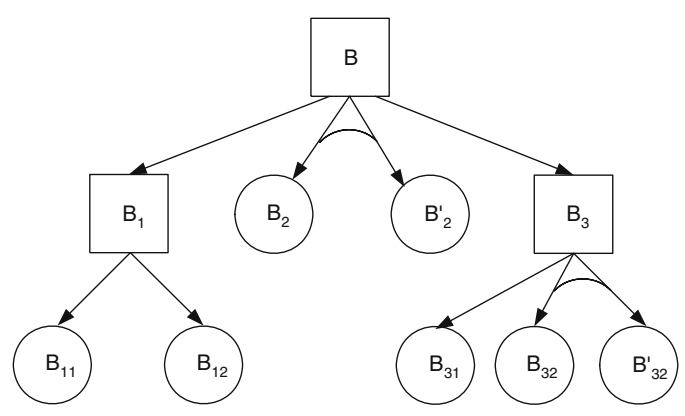

Fig. 2. Example of strategic reasoning 
$\mathcal{E}\left(B_{12}\right)=\left\{\left\{c_{12}\right\}\right\}, \mathcal{E}\left(B_{2}\right)=\left\{\left\{c_{2}\right\}\right\}, \mathcal{E}\left(B_{2}^{\prime}\right)=\left\{\left\{c_{2}^{\prime}\right\}\right\}, \mathcal{E}\left(B_{31}\right)=\left\{\left\{c_{31}\right\}\right\}, \mathcal{E}\left(\left\{B_{32}\right\}\right)$ $=\left\{\left\{c_{32}\right\}\right\}$ and $\mathcal{E}\left(\left\{B_{32}^{\prime}\right\}\right)=\left\{\left\{c_{32}^{\prime}\right\}\right\}$. The constraints $c$ and $c^{\prime}$ are defined as follows: $c=$ "the price of the car must be equal or higher than 10.000 dollars" and $c^{\prime}=$ "the buyer does not want to buy a car built in country X".

According to Definition 6 , the set of constraints associated with the strategic goals of $B(\operatorname{Str} G(B))$ is defined as follows:

$$
\begin{aligned}
& \mathcal{C} \operatorname{tr}(\{B\})=\left\{\bigcup_{\alpha \in \mathcal{C} t r(\{B(1)\})} \bigcup_{\beta \in \mathcal{C} \operatorname{tr}(\{\overline{B(1)}\})} \alpha \cup \beta\right\} \\
& \mathcal{C} t r(\{B(1)\})=\mathcal{C} t r\left(\left\{B_{1}\right\}\right)=\left\{\bigcup_{\alpha \in \mathcal{C} t r\left(\left\{B_{1}(1)\right\}\right)} \bigcup_{\beta \in \mathcal{C} \operatorname{tr}\left(\left\{\overline{B_{1}(1)}\right\}\right)} \alpha \cup \beta\right\} \\
& =\left\{\bigcup_{\alpha \in \mathcal{C} \operatorname{tr}\left(\left\{B_{11}\right\}\right)} \bigcup_{\beta \in \mathcal{C} t r\left(\left\{B_{12}\right\}\right)} \alpha \cup \beta\right\} \\
& =\left\{\bigcup_{\alpha \in \mathcal{E}\left(\left\{B_{11}\right\}\right)} \bigcup_{\beta \in \mathcal{E}\left(\left\{B_{12}\right\}\right)} \alpha \cup \beta\right\} \\
& =\left\{\bigcup_{\alpha \in\left\{\left\{c_{11}\right\}\right\}} \bigcup_{\beta \in\left\{\left\{c_{12}\right\}\right\}} \alpha \cup \beta\right\} \\
& =\left\{\left\{c_{11}, c_{12}\right\}\right\} \\
& \mathcal{C} \operatorname{tr}(\{\overline{B(1)}\})=\left\{\bigcup_{\alpha \in \mathcal{C} \operatorname{tr}(\{B(2)\})} \bigcup_{\beta \in \mathcal{C} t r(\{\overline{B(2)}\})} \alpha \cup \beta\right\} \\
& =\left\{\bigcup_{\alpha \in \mathcal{C} \operatorname{tr}\left(\left\{B_{2}, B_{2}^{\prime}\right\}\right)} \bigcup_{\beta \in \mathcal{C} t r\left(\left\{B_{3}\right\}\right)} \alpha \cup \beta\right\} \\
& =\left\{\bigcup_{\alpha \in \mathcal{C} t r\left(\left\{B_{2}\right\}\right) \cup \mathcal{C} t r\left(\left\{B_{2}^{\prime}\right\}\right)} \bigcup_{\beta \in \mathcal{C} t r\left(\left\{B_{3}\right\}\right)} \alpha \cup \beta\right\} \\
& =\left\{\bigcup_{\alpha \in \mathcal{E}\left(\left\{B_{2}\right\}\right) \cup \mathcal{E}\left(\left\{B_{2}^{\prime}\right\}\right)} \bigcup_{\beta \in \mathcal{C} \operatorname{tr}\left(\left\{B_{3}\right\}\right)} \alpha \cup \beta\right\} \\
& =\left\{\bigcup_{\alpha \in\left\{\left\{c_{2}\right\},\left\{c_{2}^{\prime}\right\}\right\}} \bigcup_{\beta \in \mathcal{C} \operatorname{tr}\left(\left\{B_{3}\right\}\right)} \alpha \cup \beta\right\} \\
& \mathcal{C} \operatorname{tr}\left(\left\{B_{3}\right\}\right)=\left\{\bigcup_{\alpha \in \mathcal{C} t r\left(\left\{B_{3}(1)\right\}\right)} \bigcup_{\beta \in \mathcal{C} t r\left(\left\{\overline{B_{3}(1)}\right\}\right)} \alpha \cup \beta\right\} \\
& =\left\{\bigcup_{\alpha \in \mathcal{C} t r\left(\left\{B_{31}\right\}\right)} \bigcup_{\beta \in \mathcal{C} t r\left(\left\{B_{32}, B_{32}^{\prime}\right\}\right)} \alpha \cup \beta\right\} \\
& =\left\{\bigcup_{\alpha \in \mathcal{C} \operatorname{tr}\left(\left\{B_{31}\right\}\right)} \bigcup_{\beta \in \mathcal{C} \operatorname{tr}\left(\left\{B_{32}\right\}\right) \cup \mathcal{C} \operatorname{tr}\left(\left\{B_{32}^{\prime}\right\}\right)} \alpha \cup \beta\right\} \\
& =\left\{\bigcup_{\alpha \in \mathcal{E}\left(\left\{B_{31}\right\}\right)} \bigcup_{\beta \in \mathcal{E}\left(\left\{B_{32}\right\}\right) \cup \mathcal{E}\left(\left\{B_{32}^{\prime}\right\}\right)} \alpha \cup \beta\right\} \\
& =\left\{\bigcup_{\alpha \in\left\{\left\{c_{31}\right\}\right\}} \bigcup_{\beta \in\left\{\left\{c_{32}\right\},\left\{c_{32}^{\prime}\right\}\right\}} \alpha \cup \beta\right\}
\end{aligned}
$$




$$
=\left\{\left\{c_{31}, c_{32}\right\},\left\{c_{31}, c_{32}^{\prime}\right\}\right\}
$$

According to the equations (3) and (4), we have:

$$
\begin{aligned}
\mathcal{C} t r(\{\overline{B(1)}\}) & =\left\{\bigcup_{\alpha \in\left\{\left\{c_{2}\right\},\left\{c_{2}^{\prime}\right\}\right\}} \bigcup_{\beta \in\left\{\left\{c_{31}, c_{32}\right\},\left\{c_{31}, c_{32}^{\prime}\right\}\right\}} \alpha \cup \beta\right\} \\
& =\left\{\left\{c_{2}, c_{31}, c_{32}\right\},\left\{c_{2}, c_{31}, c_{32}^{\prime}\right\},\left\{c_{2}^{\prime}, c_{31}, c_{32}\right\},\left\{c_{2}^{\prime}, c_{31}, c_{32}^{\prime}\right\}\right\}
\end{aligned}
$$

According to the equations (11), (2) and (5), the set of constraints associated with the goal $B$ becomes:

$$
\begin{gathered}
\operatorname{Ctr}(\{B\})=\left\{\bigcup_{\alpha \in\left\{\left\{c_{11}, c_{12}\right\}\right\}} \bigcup_{\beta \in\left\{\left\{c_{2}, c_{31}, c_{32}\right\},\left\{c_{2}, c_{31}, c_{32}^{\prime}\right\},\left\{c_{2}^{\prime}, c_{31}, c_{32}\right\},\left\{c_{2}^{\prime}, c_{31}, c_{32}^{\prime}\right\}\right\}} \alpha \cup \beta\right\} \\
=\left\{\left\{c_{11}, c_{12}, c_{2}, c_{31}, c_{32}\right\},\left\{c_{11}, c_{12}, c_{2}, c_{31}, c_{32}^{\prime}\right\},\right. \\
\left.\left\{c_{11}, c_{12}, c_{2}^{\prime}, c_{31}, c_{32}\right\},\left\{c_{11}, c_{12}, c_{2}^{\prime}, c_{31}, c_{32}^{\prime}\right\}\right\}
\end{gathered}
$$

For illustration purposes we take in the sequel a simplified case in which we have only one constraint associated with $B_{11}$ (i.e. $c_{12}=c_{2}=c_{2}^{\prime}=c_{31}=c_{32}=$ $c_{32}^{\prime}=\{\emptyset\}$ and $c_{11}=$ "the proposed car must be economic").

Furthermore, we suppose that the seller believes that the buyer often drives from Quebec to Montreal and back, and that he has a small uncomfortable car. Consequently, the seller will try to achieve the goal $B_{32}$ in order to convince the buyer that the proposed car is economic. Let us assume that during the dialogue the seller learns that the buyer does not travel regularly between Quebec and Montreal, and that he is currently working close to home. In this case, the seller is unable to convince the buyer by achieving the goal $B_{32}$, that the proposed car is has low gas consumption. Thereafter, the seller will give up the goal $B_{32}$ and will try to persist in the realization of his conversational goal which is the sale of the proposed car by using the alternative goal $B_{32}^{\prime}$. This goal aims at trying to convince the buyer that the spare parts for the proposed car are available and not expensive. If the buyer accepts the offer, then the set of constraints associated with the conversational goal $B$ is equal to $\left\{\left\{c_{11}\right\}\right\}$. In this case, we have $C(B) \cup\left\{c_{11}\right\}=\left\{c, c^{\prime}, c_{11}\right\}$ is a consistent set. Consequently, the conversational goal $B$ is achievable.

If the buyer refuses the offer unless the price is lower than 10.000 dollars, the seller will have to add the constraint $c_{32}^{\prime}$ associated with the sub-goal $B_{32}^{\prime}$. We have $c_{32}^{\prime}=$ "the price of the car must be lower than 10.000 dollars".

Since the goal $B_{32}$ is abandoned, the set of constraints including $c_{32}$ will be removed from $\mathcal{C} \operatorname{tr}(\{B\})$. The new set of constraints associated with the conversational goal $B$ becomes: $\mathcal{C} t r(\{B\})=\left\{\left\{c_{11}, c_{32}^{\prime}\right\}\right\}$. In this case, the set $C(B) \cup\left\{c_{11}, c_{32}^{\prime}\right\}=\left\{c, c^{\prime}, c_{11}, c_{32}^{\prime}\right\}$ is inconsistent. Indeed, the constraints $c$ and $c_{32}^{\prime}$ are contradictory. Consequently, the conversational goal $B$ cannot be achieved and the seller will have to change his strategy if he wants to continue the dialogue. 


\section{Conclusion}

This paper presents a formal framework based on strategic reasoning which allows agents to calculate a cognitive representation of the manner of achieving a conversational goal in terms of strategic goals. Agents' strategic goals are supported by two types of arguments: explanatory arguments which justify the choice of the goals, and realization arguments which provide the set of subgoals which are necessary to achieve these goals given a set of constraints. The second objective of this paper is to enable agents to persist in achieving their conversational goals by using alternative goals. The third objective is to provide agents by a formal method enabling them to know, at each moment, whether their goals can be achieved or not. We have illustrated each of these objectives by an example.

As future work, we integrating a rule-based planning system to the agent model. This will help in generating and revising explanatory and realization arguments. We are also interested in proposing a method enabling agents to select the most relevant and efficient strategy.

\section{References}

1. Amgoud, L., Kaci, S.: Approche basée sur l'argumentation pour la génération de buts bipolaires. In: Modèles Formels d'Intéraction (MFI 2005), Caen, May 2005, pp. 13-22 (2005)

2. Mbarki, M., Bentahar, J., Moulin, B.: Specification and Complexity of StrategicBased Reasoning Using Argumentation. In: Maudet, N., Parsons, S., Rahwan, I. (eds.) ArgMAS 2006. LNCS (LNAI), vol. 4766, pp. 142-160. Springer, Heidelberg (2007)

3. Endriss, U., Maudet, N., Sadri, F., Toni, F.: Protocol conformance for logic-based agents. In: Proceedings of the 18th International Joint Conference on Artificial Intelligence, pp. 679-684 (2003)

4. Pitt, J.: Constitutive rules for agent communication languages. In: Proceedings of the International Joint Conference on Artificial Intelligence (IJCAI 2005), Acapulco, Mexico, pp. 691-698 (2003)

5. Shapiro, S., Lesperance, Y., Levesque, H.: Goal change. In: Proceedings of the International Joint Conference on Artificial Intelligence (IJCAI 2005), Denver, CO, pp. 582-588 (2005)

6. Toni, F., Torroni, P.: Computational Logic in Multi-Agent Systems. In: Toni, F., Torroni, P. (eds.) CLIMA 2005. LNCS (LNAI), vol. 3900, Springer, Heidelberg (2006)

7. van der Hoek, W., Jamroga, W., Wooldridge, M.: A logic for strategic reasoning. In: Proceedings of the International Joint Conference on Autonomous Agents and Multi-Agent Systems (AAMAS 2005), pp. 157-164. ACM Inc., New York (2005) 